\title{
An Optimal-Order Error Estimate for the Discontinuous Galerkin Method
}

\author{
By Gerard R. Richter
}

\begin{abstract}
In this paper a new approach is developed for analyzing the discontinuous Galerkin method for hyperbolic equations. For a model problem in $R^{2}$, the method is shown to converge at a rate $O\left(h^{n+1}\right)$ when applied with $n$th degree polynomial approximations over a semiuniform triangulation, assuming sufficient regularity in the solution.
\end{abstract}

1. Introduction. We shall analyze the discontinuous Galerkin method in the context of a model hyperbolic equation:

$$
\begin{aligned}
& \alpha_{1} \frac{\partial u}{\partial x}+\alpha_{2} \frac{\partial u}{\partial y}=f(x, y), \quad(x, y) \in \Omega \subset R^{2}, \\
& u(x, y) \text { given for }(x, y) \in \Gamma_{\text {in }}(\Omega) .
\end{aligned}
$$

Here $\Gamma_{\text {in }}(\Omega)$ is the "inflow" portion of the boundary $\Gamma$ of $\Omega$, defined by

$$
\Gamma_{\text {in }}(\Omega)=\{(x, y) \in \Gamma \mid \alpha \cdot n<0\},
$$

where $n$ is the unit outer normal to $\Omega$, and $\alpha$ is assumed to be a constant vector, with $\alpha_{1}^{2}+\alpha_{2}^{2}=1$ and $\alpha_{2}>0$.

Assuming $\Omega$ is a polygon and that it has been divided into triangles, it is always possible to order the triangles $\left\{T_{1}, T_{2}, \ldots\right\}$ such that for each $k$ the domain of dependence of $T_{k}$ consists of some subset of $\Gamma_{\text {in }}(\Omega)$ and $\left\{T_{1}, \ldots, T_{k-1}\right\}$ [4]. With such an ordering, one can develop a finite element approximation in an explicit fashion, triangle by triangle. To date, the most important application of this type of finite element approach has been in solving the neutron transport equation. See, e.g., [3], [5]. Two such finite element methods have been analyzed theoretically-the discontinuous Galerkin method [2], [4], and a continuous method [1].

Our concern here is the discontinuous method, which we describe as follows: For an arbitrary domain $D$, we denote by $\mathbf{P}_{n}(D)$ the space of polynomials of degree $\leq n$ over $D$. We seek an approximate solution $u_{h}$ such that for each triangle $T$, $\left.u_{h}\right|_{T} \in \mathbf{P}_{n}(T)$ and

$$
\left(\left(u_{h}\right)_{\alpha}, v_{h}\right)-\int_{\Gamma_{\mathrm{in}}(T)}\left(u_{h}^{+}-u_{h}^{-}\right) v_{h} \alpha \cdot n=\left(f, v_{h}\right), \quad \text { all } v_{h} \in \mathbf{P}_{n}(T)
$$

where (, ) denotes the $L^{2}(T)$-inner product. Here we have used the more compact notation $\left(u_{h}\right)_{\alpha}$ for the directional derivative of $u_{h}$ with respect to $\alpha$, and, for a point $P \in \Gamma_{\text {in }}(T)$,

$$
u_{h}^{ \pm}(P) \equiv \lim _{\varepsilon \rightarrow 0+} u_{h}(P \pm \varepsilon \alpha) .
$$

Received November 17, 1986; revised February 24, 1987.

1980 Mathematics Subject Classification (1985 Revision). Primary 65M15, 65N30.

Key words and phrases. Finite element method, hyperbolic equation. 
The development of $u_{h}$ starts from an interpolant of the given initial condition along $\Gamma_{\text {in }}(\Omega)$. Note that there are two types of triangles: Those with one inflow side and those with two, hereafter referred to as type I and type II triangles. Provision in (2) for a discontinuity across the inflow, $\Gamma_{\text {in }}(T)$, allows the same space of test and trial functions, $\mathbf{P}_{n}(T)$, to be used for a triangle of either type.

The discontinuous Galerkin method was first analyzed by Lesaint and Raviart [4], who established an $L^{2}$ convergence rate of $O\left(h^{n}\right)$ for an arbitrary triangulation, where $h$ is the mesh size. Later, Johnson and Pitkäranta [2], in an analysis using variational and Fourier techniques, obtained improved error estimates in $L^{p}(\Omega)$, $p>1$, including

$$
\left\|u_{h}-u\right\|_{L^{2}(\Omega)} \leq C h^{n+1 / 2}\|u\|_{H^{n+1}(\Omega)} .
$$

In this paper we develop a new method of analysis, utilizing exact representations for $u_{h}$ on triangle boundaries. We use our approach to obtain an error estimate of the form

$$
\left\|u_{h}-u\right\|_{L^{2}(\Omega)} \leq C h^{n+1}\|u\|_{H^{n+2}(\Omega)},
$$

assuming some uniformity in the triangulation. The symbol $C$ represents a generic constant, independent of $u$ and $h$. The latter estimate indicates a higher (optimal) order of convergence while requiring an additional derivative. It arises because the error in the finite element solution is oscillatory, and is damped in type II triangles.

In Section 2 we derive relevant results applicable to single triangles of both types. In Section 3 we assemble our results into the estimate (4) for the case of a semiuniform triangulation of a periodic domain. In Section 4 we present several corroborating computational examples. We also note that the $O\left(h^{n+1}\right)$ convergence rate appears to be quite robust and can occur for irregular triangulations. In an appendix we provide a brief analysis of a modified version of the discontinuous Galerkin method in which continuity is enforced across the inflow side of type I triangles. The resulting method is slightly less costly to apply. We obtain an $O\left(h^{n}\right)$ error estimate for general meshes and show that the higher-order estimate (4) remains valid under the same uniformity conditions as for the discontinuous Galerkin method.

2. Local Properties of the Approximate Solution. In this section we establish some basic properties of the approximate solution over a single triangle. To do this, we need additional notation.

For a generic triangle $T$ we use as independent variables $s$ (parallel to $\alpha$ ) and $t$ (perpendicular to $\alpha$ ), as indicated in Figure 2.1. Triangle $T$ is described by

$$
T=\left\{(s, t) \mid s \in\left[s_{\text {in }}(t), s_{\text {out }}(t)\right], t \in\left[t_{0}, t_{1}\right]\right\},
$$

and both $\Gamma_{\text {in }}(T)$ and $\Gamma_{\text {out }}(T)$ can be parameterized by $t \in\left[t_{0}, t_{1}\right]$. We set $h \equiv t_{1}-t_{0}$ and assume that $T$ satisfies a minimum angle condition independent of $h$. We shall use several $L^{2}$ projections: An interior projection $P_{n}$ with range $\mathbf{P}_{n}(T)$, and boundary projections $P_{\text {in }}$ and $P_{\text {out }}$. If $\Gamma_{\text {in }}(T)$ consists of side(s) $\Gamma_{j}$, the corresponding interval $\left[t_{0}, t_{1}\right]$ consists of subinterval(s) $\Delta t_{j}$, and $P_{\text {in }}: L^{2}\left(\Gamma_{\text {in }}(T)\right) \rightarrow\left\{v(t)|v|_{\Delta t_{j}} \in\right.$ $\left.\mathbf{P}_{n}\left(\Delta t_{j}\right)\right\}$. We define $P_{\text {out }}$ in the same way as $P_{\text {in }}$. Note that the range of $P_{\text {in }}\left(P_{\text {out }}\right)$ is continuous only for a type I (type II) triangle. We also use the notation

$$
u_{\text {in }}(t)=\left.u\right|_{\Gamma_{\text {in }}(T)}, \quad u_{h, \text { in }}^{ \pm}(t)=\left.u_{h}^{ \pm}\right|_{\Gamma_{\text {in }}(T)}, \quad e_{\text {in }}^{-}(t)=P_{\text {in }} u_{\text {in }}(t)-u_{h, \text { in }}^{-}(t),
$$




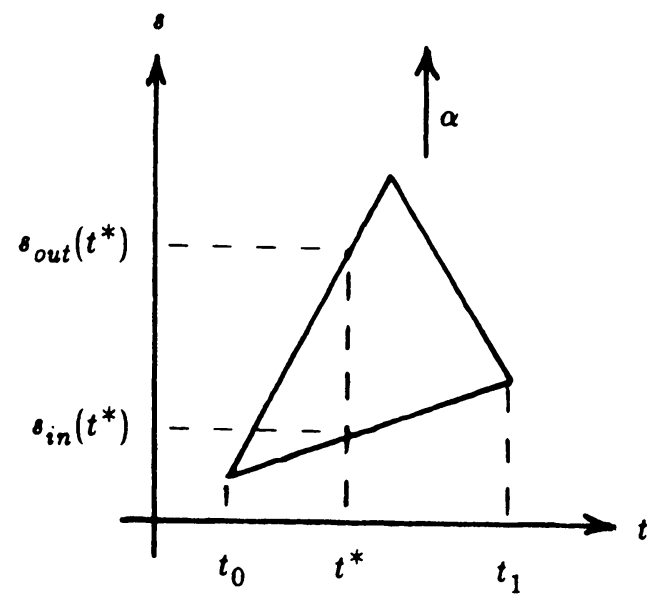

$h$

FIGURE 2.1

with an analogous set of quantities defined on $\Gamma_{\text {out }}(T)$. Finally, we denote by \|\|$_{k}$ the norm in the Sobolev space $H^{k}(T)$, with $k$ omitted as a superscript when it has value zero, and by || an $L^{2}$-norm taken with respect to $t \in\left[t_{0}, t_{1}\right]$ over the inflow or outflow boundary of $T$.

In the lemmas that follow, we establish some basic properties of $u_{h}$ over $T$.

LEMMA 2.1 (JOHNSON \& PITKÄRANTA [2]). $u_{h}$ is well defined in $T$ and has the local stability property

$$
\left\|u_{h}\right\|+h^{1 / 2}\left|u_{h}^{-}\right|_{\Gamma_{\text {out }}(T)} \leq C\left\{h^{1 / 2}\left|u_{h}^{-}\right|_{\Gamma_{\text {in }}(T)}+h\|f\|\right\} .
$$

LEMMA 2.2. For a type II triangle,

$$
\text { (i) } u_{h, \text { out }}^{-}(t)=P_{\text {out }}\left[u_{h, \text { in }}^{-}(t)+\int_{s_{\text {in }}(t)}^{s_{\text {out }}(t)} f(s, t) d s\right] \text {, }
$$

(ii) $e_{\text {out }}^{-}(t)=P_{\text {out }} e_{\text {in }}^{-}(t)$.

Proof. (i) We rewrite (2) as

(8) $\quad\left(\left(u_{h}\right)_{\alpha}, v_{h}\right)+\int_{t_{0}}^{t_{1}}\left[u_{h, \text { in }}^{+}(t)-u_{h, \text { in }}^{-}(t)\right] v_{h, \text { in }}(t) d t=\left(f, v_{h}\right), \quad v_{h} \in \mathbf{P}_{n}(T)$, where $v_{h, \text { in }}=\left.v_{h}\right|_{\Gamma_{\text {in }}(T)}$. Integration by parts yields

$$
-\left(u_{h},\left(v_{h}\right)_{\alpha}\right)+\int_{t_{0}}^{t_{1}}\left[u_{h, \text { out }}^{-}(t) v_{h, \text { out }}(t)-u_{h, \text { in }}^{-}(t) v_{h, \text { in }}(t)\right] d t=\left(f, v_{h}\right)
$$

If we take $v_{h}(s, t)=w(t)$, a polynomial of degree $n$ in $t$, then (9) becomes

$$
\int_{t_{0}}^{t_{1}}\left[u_{h, \text { out }}^{-}(t)-u_{h, \text { in }}^{-}(t)\right] w(t) d t=\int_{t_{0}}^{t_{1}}\left(\int_{s_{\text {in }}(t)}^{s_{\text {out }}(t)} f(s, t) d s\right) w(t) d t .
$$

But for a type II triangle, $w(t)$ is an arbitrary element in the range of $P_{\text {out }}$. Thus

$$
P_{\text {out }}\left[u_{h, \text { out }}^{-}(t)-u_{h, \text { in }}^{-}(t)\right]=P_{\text {out }} \int_{s_{\text {in }}(t)}^{s_{\text {out }}(t)} f(s, t) d s,
$$

which is equivalent to the desired result. 
(ii) The exact solution to (1) satisfies

$$
u_{\text {out }}(t)=u_{\text {in }}(t)+\int_{s_{\text {in }}(t)}^{s_{\text {out }}(t)} f(s, t) d s .
$$

We obtain part (ii) of the lemma by applying $P_{\text {out }}$ to (11), replacing $P_{\text {out }} u_{\text {in }}$ by $P_{\text {out }} P_{\text {in }} u_{\text {in }}$ (valid because $\operatorname{range}\left(P_{\text {out }}\right) \subset \operatorname{range}\left(P_{\text {in }}\right)$ for a type II triangle), then subtracting (6).

LEMMA 2.3. For a type I triangle,

$$
e_{\text {out }}^{-}(t)=e_{\text {in }}^{-}(t)+v(t)
$$

where

(i) $\quad P_{\text {in }} v(t)=0 \quad\left(\right.$ thus $\left.v(t) \perp e_{\text {in }}^{-}(t)\right)$,

(ii) $|v| \leq C h^{n+1 / 2}\|u\|_{n+1}$.

Proof. (i) (10) remains valid for a type I triangle, but now $w(t)$ is an arbitrary element in the range of $P_{\text {in }}$. Thus

$$
P_{\text {in }} u_{h, \text { out }}^{-}(t)=u_{h, \text { in }}^{-}(t)+P_{\text {in }} \int_{s_{\text {in }}(t)}^{s_{\text {out }}(t)} f(s, t) d s .
$$

To (11) we apply $P_{\text {in }}$, then write $P_{\text {in }} u_{\text {out }}=P_{\text {in }} P_{\text {out }} u_{\text {out }}$, and subtract (15). The result is (i).

(ii) Consider the effect of replacing $f$ in (8) by $P_{n-1} f$. [For the degenerate case $n=0$, we define $P_{-1} \equiv 0$.] The solution $u_{h}^{*}(s, t)$ to (8) would become

$$
u_{h}^{*}(s, t)=u_{h, \text { in }}^{-}(t)+\int_{s_{\text {in }}(t)}^{s} P_{n-1} f d s
$$

which has no discontinuity across the inflow boundary. This can be shown by direct substitution into (8). Moreover, the stability result (5) applied to $u_{h}^{*}-u_{h}$ yields

$$
\begin{aligned}
\left|u_{h, \text { out }}^{*}-u_{h, \text { out }}\right| & \leq C h^{1 / 2}\left\|\left(I-P_{n-1}\right) f\right\| \\
& \leq C h^{n+1 / 2}\|f\|_{n} \leq C h^{n+1 / 2}\|u\|_{n+1} .
\end{aligned}
$$

Thus,

$$
u_{h, \text { out }}^{-}(t)=u_{h, \text { in }}^{-}(t)+\int_{s_{\text {in }}(t)}^{s_{\text {out }}(t)} P_{n-1} f d s+\varepsilon, \quad|\varepsilon| \leq C h^{n+1 / 2}\|u\|_{n+1} .
$$

The exact solution can be written in an analogous form:

(19) $u_{\text {out }}(t)=u_{\text {in }}(t)+\int_{s_{\text {in }}(t)}^{s_{\text {out }}(t)} P_{n-1} f d s+\varepsilon^{\prime}, \quad \varepsilon^{\prime}=\int_{s_{\text {in }}(t)}^{s_{\text {out }}(t)}\left(I-P_{n-1}\right) f d s$

To (19) we apply $P_{\text {out }}$,

$$
P_{\text {out }} u_{\text {out }}=P_{\text {in }} u_{\text {in }}+\left(P_{\text {out }}-P_{\text {in }}\right) u_{\text {in }}+\int_{s_{\text {in }}(t)}^{s_{\text {out }}(t)} P_{n-1} f d s+P_{\text {out }} \varepsilon^{\prime}
$$


where we have used the fact that $\int_{s_{\text {in }}(t)}^{s_{\text {out }}(t)} P_{n-1} f d s$ is in the range of $P_{\text {out }}$ for a type I triangle. We then bound $\left(P_{\text {out }}-P_{\text {in }}\right) u_{\text {in }}$ and $P_{\text {out }} \varepsilon^{\prime}$ as follows:

$$
\begin{aligned}
&\left|P_{\text {out }} \varepsilon^{\prime}\right|=\max _{v_{h}(t) \in \operatorname{range}\left(P_{\text {out }}\right)} \frac{\left(\left(I-P_{n-1}\right) f, v_{h}(t)\right)}{\left|v_{h}(t)\right|} \\
& \leq \max _{v_{h}(t) \in \operatorname{range}\left(P_{\text {out }}\right)} \frac{\left\|\left(I-P_{n-1}\right) f\right\|\left\|v_{h}(t)\right\|}{\left|v_{h}(t)\right|} \\
& \leq C h^{1 / 2}\left\|\left(I-P_{n-1}\right) f\right\| \leq C h^{n+1 / 2}\|u\|_{n+1}, \\
&\left|\left(P_{\text {out }}-P_{\text {in }}\right) u_{\text {in }}\right| \leq\left|\left(I-P_{\text {out }}\right) u_{\text {in }}\right|+\left|\left(I-P_{\text {in }}\right) u_{\text {in }}\right|\quad \text { for a type I triangle }) \\
& \leq 2\left|\left(I-P_{\text {in }}\right) u_{\text {in }}\right| \quad\left(\text { since range }\left(P_{\text {in }}\right) \subset \operatorname{range}\left(P_{\text {out }}\right)\right. \\
& \leq \\
& \leq \\
& \leq C h^{n+1 / 2}\|u\|_{n+1} .
\end{aligned}
$$

Subtracting (18) from (20) and applying the above bounds, we obtain (ii).

The preceding lemmas lead to an $O\left(h^{n+1 / 2}\right)$ error estimate for $u_{h}$ over an arbitrary triangulation of $\Omega$. For, on a type I triangle, via (12)-(14),

$$
\left|e_{\text {out }}^{-}\right|^{2}=\left|e_{\text {in }}^{-}\right|^{2}+|v|^{2} \leq\left|e_{\text {in }}^{-}\right|^{2}+C h^{2 n+1}\|u\|_{n+1}^{2},
$$

and, on a type II triangle, via (7),

$$
\left|e_{\text {out }}^{-}\right|^{2} \leq\left|e_{\text {in }}^{-}\right|^{2} \text {. }
$$

Assuming that, at a certain point, $u_{h}$ has been developed in $\Omega_{j} \subseteq \Omega$, we sum (21) and (22) over all the triangles of $\Omega_{j}$ to obtain

$$
\left|e_{\text {out }}^{-}\right|_{\Gamma_{\text {out }}\left(\Omega_{j}\right)}^{2} \leq\left|e_{\text {in }}^{-}\right|_{\Gamma_{\text {in }}\left(\Omega_{j}\right)}^{2}+C h^{2 n+1}\|u\|_{n+1, \Omega_{j}}^{2} .
$$

Thus, if $\left.u_{h}^{-}\right|_{\Gamma_{\text {in }}(\Omega)}$ is chosen to be a standard interpolant of the given initial data,

$$
\left|e_{h, \text { out }}^{-}\right| \Gamma_{\text {out }}\left(\Omega_{j}\right) \leq C h^{n+1 / 2}\|u\|_{n+1, \Omega_{j}},
$$

which implies

$$
\left|u-u_{h}^{-}\right|_{\Gamma_{\text {out }}\left(\Omega_{j}\right)} \leq C h^{n+1 / 2}\|u\|_{n+1, \Omega_{j}} .
$$

An analogous interior estimate,

$$
\left\|u-u_{h}\right\|_{\Omega_{j}} \leq C h^{n+1 / 2}\|u\|_{n+1, \Omega_{j}}
$$

can then be obtained by a suitable application of Lemma 2.1. This result has already been obtained by Johnson and Pitkäranta [2] using variational and Fourier analysis techniques. We shall use our approach to show that $u_{h}$ converges at a rate $O\left(h^{n+1}\right)$ under certain conditions. The key observation is that (22) fails to exploit any potential damping of the error as it is projected across a type II triangle via (7). We proceed with the development of a mechanism which accounts for this.

Consider the situation where there are two adjoining type I triangles $T$ and a translate $T_{1}=\{(s+k, t+h) \mid(s, t) \in T\}$, as shown in Figure 2.2. For a function $w$ defined on $T \cup T_{1}$, we define

$$
\Delta w(s, t) \equiv w(s+k, t+h)-w(s, t), \quad(s, t) \in T .
$$




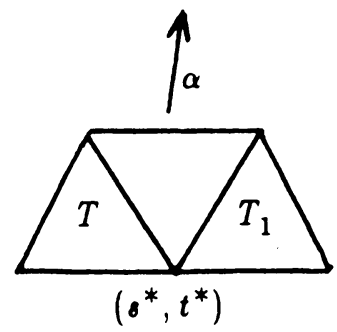

FIGURE 2.2

Via superposition, we may regard $\Delta u$ on $T$ as the solution of

$$
\begin{aligned}
& (\Delta u)_{\alpha}=\Delta f, \\
& \Delta u=\Delta u_{\text {in }} \text { on } \Gamma_{\text {in }}(T),
\end{aligned}
$$

and $\Delta u_{h}$ as its finite element approximation. Thus from Lemma 2.3, we infer that

$$
\Delta e_{\text {out }}^{-}(t)=\Delta e_{\text {in }}^{-}(t)+\Delta v(t)
$$

where

$$
|\Delta v| \leq C h^{n+1 / 2}\|\Delta u\|_{n+1} .
$$

Let us denote by $\left(s^{*}, t^{*}\right)$ the coordinates of the common vertex of $T$ and $T_{1}$. Then

$$
\begin{aligned}
\|\Delta u\|_{n+1}^{2} & =\sum_{0 \leq|k| \leq n+1} \|\left(D^{k} u(s+k, t+h)-D^{k} u\left(s^{*}, t^{*}\right)\right) \\
& \leq 2 \sum_{0 \leq|k| \leq n+1}\left\{\| D^{k} u(s, t)-D^{k} u(s, t)-D^{k} u\left(s^{*}, t^{*}\right)\right) \|^{2} \\
& \left.\leq C h^{2} \sum_{1 \leq|k| \leq n+2}\left\|D^{k} u\right\|_{T \cup T_{1}}^{2}\right\}^{2} \\
& \leq C T^{2}\|u\|_{n+2, T \cup T_{1}}^{2} .
\end{aligned}
$$

We summarize as follows:

LEMMA 2.4. For a type I triangle $T$ with an adjoining translate $T_{1}$ as in Figure 2.2 ,

$$
\Delta e_{\text {out }}^{-}(t)=\Delta e_{\text {in }}^{-}(t)+\Delta v(t)
$$

where

$$
|\Delta v(t)| \leq C h^{n+3 / 2}\|u\|_{n+2, T \cup T_{1}} .
$$

3. Error Estimate for a Semiuniform Triangulation. We now extend the results of Section 2 to an entire triangulation, constructed as follows. We take $\Omega \equiv[0,2 \pi] \times[0,1]$ as the domain of $(1)$ and assume periodicity with respect to the first variable, so that only an initial condition $u(x, 0)$ is needed. We partition $\Omega$ into layers

$$
S_{j}=\left\{(x, y) \in[0,2 \pi] \times\left[y_{j}, y_{j+1}\right]\right\}, \quad j=0, \ldots, m-1,
$$

where $0=y_{0}<y_{1}<y_{2}<\cdots<y_{m}=1$. We then divide each layer into "halflayers" of congruent type I and type II triangles, as shown in Figure 3.1. The finite element solution can be thought of as developing a half-layer at a time, and in 


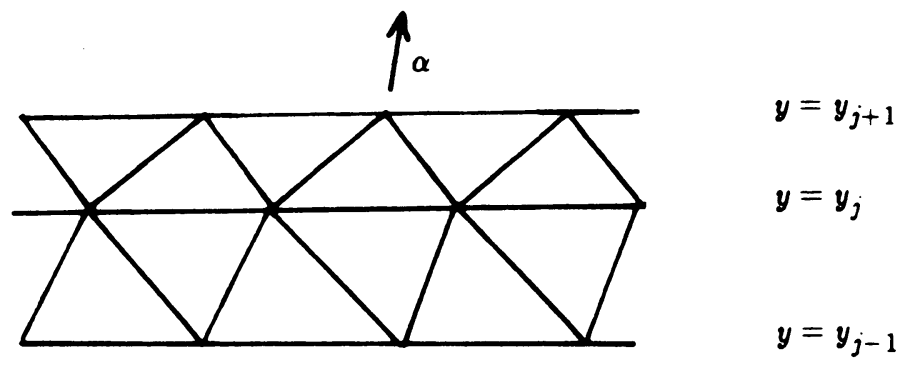

FIGURE 3.1

parallel over the triangles comprising each half-layer. Note that all the triangles in this triangulation of $\Omega$ have the same horizontal side length. We shall consider a family of such triangulations satisfying two additional conditions:

H1. The angles of all triangles of $\Omega$ are uniformly bounded away from zero.

H2. $|\alpha \cdot n|$ is uniformly bounded away from zero.

We also need some new notation. We denote by $P_{j}$ the $L^{2}$-projection into (in general, discontinuous) piecewise $n$th degree polynomials in $t$ with respect to the grid points along $y=y_{j}$, and by $Q_{j}$ the analogous projection into piecewise constants at level $j$. We then define

$$
u_{j}(t)=\left.u\right|_{y=y_{j}}, \quad u_{h, j}^{-}(t)=\left.u_{h}^{-}\right|_{y=y_{j}}, \quad e_{j}^{-}(t)=P_{j} u_{j}(t)-u_{h, j}^{-}(t),
$$

and, for an arbitrary function $z_{j}(t)$ defined along $y=y_{j}, \Delta z_{j}(t) \equiv z_{j}(t+h)-$ $z_{j}(t)$. In general, a subscript $j$, as in $z_{j}(t)$, will denote a piecewise $n$th degree polynomial with respect to the grid points along level $y_{j}$. Similarly, $z_{j+1 / 2}$ will signify a piecewise $n$th degree polynomial with respect to the outflow boundary of the type I portion of $S_{j}$. Finally, we denote by $\left|z_{j}\right|$ the $L^{2}$-norm of $z_{j}(t)$ over level j.

By applying Lemmas 2.2-2.4 to the $j$ th layer we obtain

\section{LEMMA 3.1. There holds}

$$
e_{j+1}^{-}(t)=P_{j+1}\left(e_{j}^{-}(t)+v_{j+1 / 2}(t)\right),
$$

where

$$
\text { (i) } \quad\left|v_{j+1 / 2}\right| \leq C h^{n+1 / 2}\|u\|_{n+1, S_{j}} \text {, }
$$$$
\text { (ii) } Q_{j} v_{j+1 / 2}=0 \text {, }
$$$$
\text { (iii) }\left|\Delta v_{j+1 / 2}\right| \leq C h^{n+3 / 2}\|u\|_{n+2, S_{j}} \text {. }
$$

Our strategy for establishing an $O\left(h^{n+1}\right)$ convergence rate will involve showing that the influence of $v_{j+1 / 2}$ declines exponentially as the solution advances beyond the $j$ th layer. At a given level, the cumulative effect of all previous $v_{j+1 / 2}$ 's will then be that of a geometrically decaying sum. Observe that if $z_{j}(t)$ is a nonvanishing piecewise polynomial at level $j$ satisfying $Q_{j} z_{j}=0$ and $\Delta z_{j}=0$ (implying that $z_{j}(t)$ has period $h)$, then $z_{j+1} \equiv P_{j+1} z_{j}$ satisfies $\left|z_{j+1}\right|<\left|z_{j}\right|$. Further, $Q_{j+1} z_{j+1}=0$ and $\Delta z_{j+1}=0$, so subsequent projection onto a new level will produce additional damping. Our situation is similar, although complicated by the fact that $\left|\Delta v_{j+1 / 2}\right|$, while small in relation to $\left|v_{j+1 / 2}\right|$, is in general nonzero. In the following lemma, 


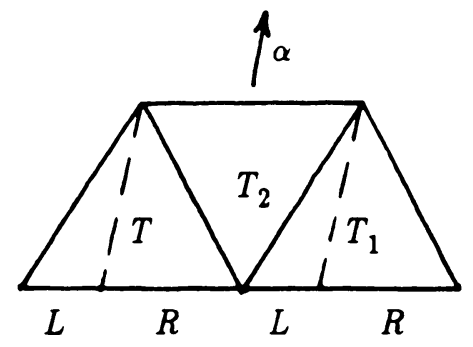

FIGURE 3.2

we maintain $e_{j}^{-}(t)$ inductively in the form $e_{j}^{-}(t)=w_{j}(t)+r_{j}(t)$, where $Q_{j} w_{j}=0$, and monitor the growth of $w_{j}, \Delta w_{j}$ and $r_{j}$.

LEMMA 3.2. Suppose $e_{j}^{-}(t)$ has a representation

$$
e_{j}^{-}(t)=w_{j}(t)+r_{j}(t)
$$

where $Q_{j} w_{j}=0$. Then, by $(26)$ and $(28), e_{j+1}^{-}(t)$ can be represented as

$$
e_{j+1}^{-}(t)=w_{j+1}(t)+r_{j+1}(t),
$$

where

$$
\text { (i) } Q_{j+1} w_{j+1}=0 \text {, }
$$$$
\text { (ii) }\left|w_{j+1}\right| \leq \lambda\left|w_{j}\right|+\left|v_{j+1 / 2}\right| \text {, }
$$$$
\text { (iii) }\left|\Delta w_{j+1}\right| \leq \lambda\left|\Delta w_{j}\right|+\left|\Delta v_{j+1 / 2}\right| \text {, }
$$$$
\text { (iv) }\left|r_{j+1}\right| \leq\left|r_{j}\right|+\left|\Delta v_{j+1 / 2}\right|+\left|\Delta w_{j}\right| \text {, }
$$

where $\lambda<1$ is a constant, independent of $h$.

Proof. By projecting the grid points along $y=y_{j+1}$ backward to $y=y_{j}$ along characteristics, we partition the subintervals of $y=y_{j}$ into left and right sections, as indicated in Figure 3.2 by $L$ and $R$. We define

$$
\begin{aligned}
& \bar{w}_{j+1 / 2}(t)=\left\{\begin{array}{lr}
w_{j}(t), & t \in L, \\
w_{j}(t+h), & t \in R,
\end{array}\right. \\
& \bar{v}_{j+1 / 2}(t)=\left\{\begin{array}{lr}
v_{j+1 / 2}(t), & t \in L, \\
v_{j+1 / 2}(t+h), & t \in R .
\end{array}\right.
\end{aligned}
$$

Note that

$$
\left|\bar{w}_{j+1 / 2}-w_{j}\right| \leq\left|\Delta w_{j}\right|, \quad\left|\bar{v}_{j+1 / 2}-v_{j+1 / 2}\right| \leq\left|\Delta v_{j+1 / 2}\right| .
$$

We set

$$
\begin{gathered}
w_{j+1} \equiv P_{j+1}\left(\bar{w}_{j+1 / 2}+\bar{v}_{j+1 / 2}\right), \\
r_{j+1} \equiv P_{j+1}\left(r_{j}+w_{j}-\bar{w}_{j+1 / 2}+v_{j+1 / 2}-\bar{v}_{j+1 / 2}\right) .
\end{gathered}
$$

Using (26) and the assumed representation for $e_{j}^{-}$, we obtain

$$
e_{j+1}^{-}=P_{j+1}\left(w_{j}+r_{j}+v_{j+1 / 2}\right)=w_{j+1}+r_{j+1} .
$$

We now prove (i)-(iv). 
(i) We regard $\bar{w}_{j+1 / 2}(t)$ as being defined on the inflow to the type II portion of $S_{j}$ (its discontinuities lie at the grid points of this "half-level"). Note that in Figure $3.2,\left.\bar{w}_{j+1 / 2}\right|_{\Gamma_{\text {in }}\left(T_{2}\right)}$ arises from $\left.w_{j}\right|_{\Gamma_{\text {in }}\left(T_{1}\right)}$ by "breaking off" the right section of the latter and "appending" it on the left. Thus

$$
\int_{\Gamma_{\text {in }}\left(T_{2}\right)} \bar{w}_{j+1 / 2}(t) d t=\int_{\Gamma_{\text {in }}\left(T_{1}\right)} w_{j}(t) d t,
$$

and $Q_{j} w_{j}=0$ implies that $Q_{j+1} \bar{w}_{j+1 / 2}=0$. Analogously, one may show that $Q_{j+1} \bar{v}_{j+1 / 2}=0$. Thus $Q_{j+1} w_{j+1}=Q_{j+1}\left(\bar{w}_{j+1 / 2}+\bar{v}_{j+1 / 2}\right)=0$.

(ii) If $\left.\bar{w}_{j+1 / 2}(t)\right|_{\Gamma_{\text {In }}\left(T_{2}\right)}$ is a polynomial (as opposed to a piecewise polynomial), then it must vanish identically. For, if $\left.\bar{w}_{j+1 / 2}(t)\right|_{\Gamma_{\mathrm{in}}\left(T_{2}\right)}$ is a polynomial, then

$$
\xi_{j}(t) \equiv \begin{cases}w_{j}(t), & t \in \Gamma_{\text {in }}\left(T_{1}\right), \\ w_{j}(t+h), & t \in \Gamma_{\text {in }}(T)\end{cases}
$$

must be a polynomial on $\Gamma_{\mathrm{in}}(T) \cup \Gamma_{\mathrm{in}}\left(T_{1}\right)$, with $\left.\xi_{j}\right|_{\Gamma_{\mathrm{in}}(T)}$ and $\left.\xi_{j}\right|_{\Gamma_{\text {in }}\left(T_{1}\right)}$ periodic images of each other. This is impossible unless $\xi_{j} \equiv$ constant. But $Q_{j} w_{j}=0$, so $\xi_{j} \equiv 0$, which implies that $\left.\bar{w}_{j+1 / 2}(t)\right|_{\Gamma_{\text {in }}\left(T_{2}\right)} \equiv 0$. Hence $\left.\bar{w}_{j+1 / 2}(t)\right|_{\Gamma_{\text {in }}\left(T_{2}\right)}$, if nonzero, is not a polynomial and will therefore undergo a decrease in norm when projected onto $\Gamma_{\text {out }}\left(T_{2}\right)$. Thus,

$$
\left|P_{j+1} \bar{w}_{j+1 / 2}\right|_{\Gamma_{\text {out }}\left(T_{2}\right)} \leq \lambda\left|\bar{w}_{j+1 / 2}\right|_{\Gamma_{\text {in }}\left(T_{2}\right)}=\lambda\left|w_{j}\right|_{\Gamma_{\text {in }}\left(T_{1}\right)}, \quad \lambda<1 .
$$

Moreover, the constant $\lambda$ may be taken to be uniformly less than one and independent of $h$, in view of Assumption H2. Application of (37) over the entire layer then yields

$$
\left|P_{j+1} \bar{w}_{j+1 / 2}\right| \leq \lambda\left|w_{j}\right|
$$

which, with (35) and the fact that

$$
\left|P_{j+1} \bar{v}_{j+1 / 2}\right| \leq\left|\bar{v}_{j+1 / 2}\right|=\left|v_{j+1 / 2}\right|,
$$

proves (ii).

(iii) This is analogous to (ii) and follows in the same way via superposition.

(iv) This follows from (36) and (34).

The solutions of $(30)-(32)$ are

$$
\begin{aligned}
& \left|w_{j}\right| \leq \lambda^{j}\left|w_{0}\right|+\sum_{k=0}^{j-1} \lambda^{k}\left|v_{j-k-1 / 2}\right| \\
& \left|\Delta w_{j}\right| \leq \lambda^{j}\left|\Delta w_{0}\right|+\sum_{k=0}^{j-1} \lambda^{k}\left|\Delta v_{j-k-1 / 2}\right| \\
& \left|r_{j}\right| \leq\left|r_{0}\right|+\sum_{k=0}^{j-1}\left(\left|\Delta v_{k+1 / 2}\right|+\left|\Delta w_{k}\right|\right)
\end{aligned}
$$

Thus,

$$
\begin{aligned}
& \left|w_{j}\right| \leq \lambda^{j}\left|w_{0}\right|+\frac{1}{1-\lambda} \max _{0 \leq k \leq j-1}\left|v_{k+1 / 2}\right|, \\
& \left|\Delta w_{j}\right| \leq \lambda^{j}\left|\Delta w_{0}\right|+\frac{1}{1-\lambda} \max _{0 \leq k \leq j-1}\left|\Delta v_{k+1 / 2}\right|, \\
& \left|r_{j}\right| \leq\left|r_{0}\right|+\frac{1}{1-\lambda}\left|\Delta w_{0}\right|+\frac{2-\lambda}{1-\lambda} j \max _{0 \leq k \leq j-1}\left|\Delta v_{k+1 / 2}\right| .
\end{aligned}
$$


Summation of (38) and (39) yields

$$
\begin{aligned}
\left|e_{j}^{-}\right| \leq & \lambda^{j}\left|w_{0}\right|+\frac{1}{1-\lambda}\left|\Delta w_{0}\right|+\left|r_{0}\right| \\
& +\frac{1}{1-\lambda}\left\{\max _{0 \leq k \leq j-1}\left|v_{k+1 / 2}\right|+(2-\lambda) j \max _{0 \leq k \leq j-1}\left|\Delta v_{k+1 / 2}\right|\right\} .
\end{aligned}
$$

We now set

$$
w_{0}=\left(I-Q_{0}\right) e_{0}^{-}, \quad r_{0}=Q_{0} e_{0}^{-}
$$

so that, at level 0 , the premise of Lemma 3.2 is satisfied. Then

$$
\left|w_{0}\right| \leq\left|e_{0}^{-}\right|, \quad\left|r_{0}\right| \leq\left|e_{0}^{-}\right|, \quad\left|\Delta w_{0}\right| \leq 2\left|e_{0}^{-}\right| .
$$

Substituting these bounds into (40) and applying (27) and (29), we obtain

LEMMA 3.3. The error at level $j$ satisfies

$$
\begin{aligned}
\left|e_{j}^{-}\right| \leq \frac{1}{1-\lambda}\left\{4\left|e_{0}^{-}\right|+C h^{n+1 / 2}(\right. & \max _{0 \leq k \leq j-1}\|u\|_{n+1, S_{k}} \\
& \left.\left.+j h \max _{0 \leq k \leq j-1}\|u\|_{n+2, S_{k}}\right)\right\} .
\end{aligned}
$$

We now make the regularity assumption

H3. $\|u\|_{n+2, S_{j}} \leq C \sqrt{h}\|u\|_{n+2, \Omega}$,

implying, in a sense, that $\|u\|_{n+2, \Omega}$ is distributed uniformly over the layers of $\Omega$. We also assume that the finite element solution at level 0 is a standard interpolant of the exact initial condition, so that

$$
\left|e_{0}^{-}\right| \leq C h^{n+1 / 2}\|u\|_{n+1, S_{0}} .
$$

Using the fact that there are $O\left(h^{-1}\right)$ layers in all (implied by Assumption H1), we conclude that

$$
\max _{0 \leq j \leq m}\left|e_{j}^{-}\right| \leq C h^{n+1}\|u\|_{n+2, \Omega}
$$

and, as a result,

$$
\max _{0 \leq j \leq m}\left|u_{h}^{-}-u\right|_{y=y_{j}} \leq C h^{n+1}\|u\|_{n+2, \Omega}
$$

To obtain an interior estimate of the error in $u_{h}$, we write (2) in the form

$$
\left(\left(u_{h}\right)_{\alpha}, v_{h}\right)-\int_{\Gamma_{\text {in }}(T)}\left(u_{h}^{+}-u_{h}^{-}\right) v_{h} \alpha \cdot n=\left(u_{\alpha}, v_{h}\right),
$$

and note that for the standard continuous $n$th degree piecewise polynomial interpolant $u_{I} \approx u$,

$$
\left(\left(u_{I}\right)_{\alpha}, v_{h}\right)-\int_{\Gamma_{\mathrm{in}}(T)}\left(u_{I}^{+}-u_{I}^{-}\right) v_{h} \alpha \cdot n=\left(\left(u_{I}\right)_{\alpha}, v_{h}\right) .
$$

Subtracting, we obtain

$$
\left(\left(u_{h}-u_{I}\right)_{\alpha}, v_{h}\right)-\int_{\Gamma_{\text {in }}(T)}\left[\left(u^{h}-u_{I}\right)^{+}-\left(u_{h}-u_{I}\right)^{-}\right] v_{h} \alpha \cdot n=\left(\left(u-u_{I}\right)_{\alpha}, v_{h}\right) .
$$

Application of the local stability result (5) over the triangles in $S_{j}$ then yields

$$
\begin{aligned}
\left\|u_{h}-u_{I}\right\|_{S_{j}} & \leq C\left\{\sqrt{h}\left|u_{h}^{-}-u_{I}^{-}\right|_{y=y_{j}}+h\left\|\left(u-u_{I}\right)_{\alpha}\right\|_{S_{j}}\right\} \\
& \leq C\left\{h^{n+3 / 2}\|u\|_{n+2, \Omega}+h^{n+1}\|u\|_{n+1, S_{j}}\right\}
\end{aligned}
$$


using (42) and relevant approximation properties of $u_{I}$. Thus,

$$
\begin{aligned}
\left\|u_{h}-u_{I}\right\|_{\Omega}^{2} & \leq C\left\{m h^{2 n+3}\|u\|_{n+2, \Omega}^{2}+h^{2 n+2}\|u\|_{n+1, \Omega}^{2}\right\} \\
& \leq C h^{2 n+2}\|u\|_{n+2, \Omega}^{2}
\end{aligned}
$$

and

$$
\left\|u_{h}-u\right\|_{\Omega} \leq\left\|u_{h}-u_{I}\right\|_{\Omega}+\left\|u_{I}-u\right\|_{\Omega} \leq C h^{n+1}\|u\|_{n+2, \Omega}
$$

We summarize as follows:

THEOREM 3.1. Under Assumptions H1-H3, the discontinuous Galerkin approximation $u_{h}$ satisfies

$$
\left\|u_{h}-u\right\|_{\Omega}+\max _{0 \leq j \leq m}\left|u_{h}^{-}-u\right|_{y=y_{j}} \leq C h^{n+1}\|u\|_{n+2, \Omega}
$$

We conclude this section with several remarks. We note first that the $O\left(h^{n+1}\right)$ error estimate is a result of the damping which accompanies projection of the error from the grid at one level to the interlacing grid at the next level. Specifically, the fact that $Q_{j} w_{j}=0$ in the representation $e_{j}^{-}(t)=w_{j}(t)+r_{j}(t)$ implies that $w_{j}$ is highly oscillatory and hence damped by $P_{j+1}$. Our computational experimentation indicates that the $O\left(h^{n+1}\right)$ convergence rate is not confined to the assumptions made in our analysis, and that it can occur even for irregular triangulations. We believe that the basic mechanism involved is the damping of an oscillatory error via (7). Some of our assumptions were motivated by ease of exposition rather than necessity, e.g., that of a periodic domain. Also, $\mathrm{H} 2$ is not essential, for in the limiting case where $\alpha \cdot n=0$ in a layer $S_{j}, P_{j+1}=P_{j}$ and (26) becomes $e_{j+1}^{-}(t)=e_{j}^{-}(t)$ (because Lemmas 2.2-2.4 imply that $P_{j} v_{j+1 / 2}=0$ ). Thus, there is no growth in the error over such a layer.

4. Computational Results. We use as a test problem

$$
\frac{1}{2} \frac{\partial u}{\partial x}+\frac{\sqrt{3}}{2} \frac{\partial u}{\partial y}=0, \quad x \in(-\infty, \infty), t>0,
$$

\begin{tabular}{|c|c|c|c|c|}
\hline \multicolumn{5}{|c|}{$L^{2} e$} \\
\hline$\underline{\Delta x}$ & error at $y=1$ & $\underline{\text { ratio }}$ & error at $y=2$ & $\underline{\text { ratio }}$ \\
\hline 1 & .5338 & $* * * *$ & .5803 & $* * * *$ \\
\hline .5 & .3383 & 1.58 & .4166 & 1.39 \\
\hline .25 & .2156 & 1.57 & .3009 & 1.38 \\
\hline .125 & .1267 & 1.70 & .1969 & 1.53 \\
\hline .0625 & $.6989(-1)$ & 1.81 & .1175 & 1.68 \\
\hline .03125 & $.3693(-1)$ & 1.89 & $.6524(-1)$ & 1.80 \\
\hline 015625 & $.1902(-1)$ & 1.94 & $.3458(-1)$ & 1.89 \\
\hline
\end{tabular}

with $u(x, 0)$ a cubic B-spline with knots at $x=-1,-.5,0, .5,1.0$ and maximum value 1 at $x=0$. The exact solution is thus the same cubic $B$-spline, propagated along characteristics at an angle of $60^{\circ}$ with the $x$-axis. We triangulate $\Omega$ by means

\section{TABLE 4.1}


of a uniform set of right isosceles triangles with their hypotenuses parallel to the $x$-axis, producing a configuration similar to that depicted in Figure 3.1. Table 4.1 shows the $L^{2}$ error in the piecewise constant discontinuous Galerkin approximation at $y=1$ and $y=2$, as well as ratios of successive values of the error as the subinterval size $\Delta x$ is repeatedly halved. The exact solution has sufficient smoothness to make the estimate (43) applicable, and the results are consistent with the predicted $O(h)$ rate of convergence.

Table 4.2 shows the analogous computational results for the case of piecewise linear approximation. Again, the optimal order of convergence is occurring, as predicted.

\begin{tabular}{|c|c|c|c|c|}
\hline \multicolumn{5}{|c|}{ TABLE 4.2} \\
\hline \multicolumn{5}{|c|}{$L^{2}$ errors; piecewise linear approximation } \\
\hline$\Delta x$ & error at $y=1$ & ratio & error at $y=2$ & $\underline{\text { ratio }}$ \\
\hline 1 & .2597 & $* * * *$ & .2650 & $* * * *$ \\
\hline .5 & $.7253(-1)$ & 3.58 & .1061 & 2.50 \\
\hline .25 & $.2109(-1)$ & 3.44 & $.2598(-1)$ & 4.08 \\
\hline .125 & $.5164(-2)$ & 4.08 & $.5784(-2)$ & 4.49 \\
\hline .0625 & $.1279(-2)$ & 4.04 & $.1351(-2)$ & 4.28 \\
\hline .03125 & $.3173(-3)$ & 4.03 & $.3248(-3)$ & 4.16 \\
\hline .015625 & $.7982(-4)$ & 4.02 & $.7977(-4)$ & 4.07 \\
\hline
\end{tabular}

To illustrate that the optimal order of convergence may occur even for nonuniform triangulations, we randomly perturbed each triangle vertex, the $x$ coordinate by as much as $.15 \Delta x$, and a proportionate amount for the $y$ coordinate, and repeated the experiment. The $L^{2}$ errors in the piecewise constant and piecewise linear discontinuous Galerkin approximations are shown in Tables 4.3 and 4.4. They again indicate an optimal order of convergence. We have not conducted a thorough study of the conditions under which the optimal order of convergence occurs, but this phenomenon appears to be quite robust.

TABLE 4.3

$L^{2}$ errors; piecewise constant approximation; randomly perturbed grid points

\begin{tabular}{rlcccc}
$\underline{\Delta x}$ & error at $y=1$ & & ratio & error at $y=2$ & $\underline{\text { ratio }}$ \\
\cline { 5 - 6 } 1 & .5382 & $* * * *$ & .5867 & $* * * *$ \\
.5 & .3402 & 1.58 & .4153 & 1.43 \\
.25 & .2212 & 1.54 & .2964 & 1.40 \\
.125 & .1238 & 1.79 & .1946 & 1.52 \\
.0625 & $.6906(-1)$ & 1.79 & .1152 & 1.69 \\
.03125 & $.3631(-1)$ & 1.90 & $.6367(-1)$ & 1.81 \\
.015625 & $.1866(-1)$ & 1.95 & $.3365(-1)$ & 1.89
\end{tabular}


TABLE 4.4

$L^{2}$ errors; piecewise linear approximations; randomly perturbed grid points

$\underline{\Delta x}$ error at $y=1 \quad \underline{\text { ratio }}$ error at $y=2 \quad \underline{\text { ratio }}$

$\begin{array}{rlclc}1 & .2412 & * * * * & .2497 & * * * * \\ .5 & .7649(-1) & 3.15 & .1040 & 2.40 \\ .25 & .2074(-1) & 3.69 & .3198(-1) & 3.25 \\ .125 & .6473(-2) & 3.20 & .6124(-1) & 5.22 \\ .0625 & .1410(-2) & 4.59 & .1498(-2) & 4.09 \\ .03125 & .3615(-3) & 3.90 & .3663(-3) & 4.09 \\ .015625 & .8889(-4) & 4.07 & .9655(-4) & 3.79\end{array}$

Appendix. We briefly consider a variant of the discontinuous Galerkin method in which $u_{h}$ is defined by (2) in type II triangles, and by

$$
\begin{aligned}
& \left(\left(u_{h}\right)_{\alpha}, v_{h}\right)=\left(f, v_{h}\right), \quad \text { all } v_{h} \in \mathbf{P}_{n-1}(T), \\
& u_{h}^{+}=u_{h}^{-} \text {on } \Gamma_{\text {in }}(T)
\end{aligned}
$$

in type I triangles. We assume $n \geq 1$ so that the inner product conditions in (45) are nonvacuous. Since the approximate solution $u_{h}$ is now continuous along the inflow to type I triangles, it has fewer degrees of freedom; thus type I triangles yield smaller linear algebraic systems. This could produce a possibly significant saving in computational expense for small values of $n$. For example, with $n=2$, (45) reduces to a linear system of order 3 vs. 6 for the discontinuous Galerkin method.

To obtain a closed form representation for $u_{h}$ in a type I triangle $T$, observe that the inner product conditions in (45) are equivalent to

$$
\left(u_{h}\right)_{\alpha}=P_{n-1} f \text {. }
$$

Integration along characteristics then yields the unique solution

$$
u_{h}(s, t)=u_{h, \text { in }}^{-}(t)+\int_{s_{\text {in }}(t)}^{s} P_{n-1} f d s, \quad(s, t) \in T .
$$

As noted in the proof of Lemma 2.3, the discontinuous Galerkin method will produce the same approximate solution as the above (cf. (16)) if $\left(I-P_{n-1}\right) f=0$ in $T$. In particular, for the homogeneous version of $(1), f \equiv 0$, there is no difference between the two solutions.

To analyze the error in the modified scheme, we evaluate (46) at $s_{\text {out }}(t)$,

$$
u_{h, \text { out }}^{-}(t)=u_{h, \text { in }}^{-}(t)+\int_{s_{\text {in }}(t)}^{s_{\text {out }}(t)} P_{n-1} f d s,
$$

then apply $P_{\text {out }}$ to (11):

$$
P_{\text {out }} u_{\text {out }}(t)=P_{\text {in }} u_{\text {in }}(t)+\left(P_{\text {out }}-P_{\text {in }}\right) u_{\text {in }}(t)+P_{\text {out }} \int_{s_{\text {in }}(t)}^{s_{\text {out }}(t)} f d s .
$$

Subtracting (48) from (47), we obtain

$$
e_{\text {out }}^{-}(t)=e_{\text {in }}^{-}(t)+v(t)
$$


where

$$
v(t)=\left(P_{\text {in }}-P_{\text {out }}\right) u_{\text {in }}(t)+P_{\text {out }} \int_{s_{\text {in }}(t)}^{s_{\text {out }}(t)}\left(P_{n-1}-I\right) f d s .
$$

As in Lemma 2.3,

$$
|v| \leq C h^{n+1 / 2}\|u\|_{n+1, T}
$$

However, in place of (13), we have a weaker orthogonality condition,

$$
P_{\text {in }}^{*} v(t)=0,
$$

where $P_{\text {in }}^{*}$ is defined to be the $L^{2}$ projection into polynomials of degree $\leq n-1$ on $\Gamma_{\text {in }}(T)$. [In obtaining the above relation, we have used the fact that, for a polynomial $w(t)$ of degree $\leq n-1,\left(w(t), \int_{s_{\text {in }}(t)}^{s_{\text {out }}(t)}\left(I-P_{n-1}\right) f d s\right)=$ $\int_{T} w\left(I-P_{n-1}\right) f=0$.] As a result of the reduced orthogonality, we have only

$$
\left|e_{\text {out }}^{-}\right|^{2} \leq(1+O(h))\left|e_{\text {in }}^{-}\right|^{2}+C h^{2 n}\|u\|_{n+1, T}^{2}
$$

(using the arithmetic-geometric mean inequality) instead of (21). When combined with (22), inequality (50) leads to an $O\left(h^{n}\right)$ estimate for the $L^{2}$ error in $u_{h}$.

However, the $O\left(h^{n+1}\right)$ estimate (43) remains applicable to the modified method under the same assumptions as for the fully discontinuous method. This follows from the observation that Lemma 2.4 is still valid, and the fact that the orthogonality condition (49) is sufficient to make $Q_{j} v_{j+1 / 2}=0$ (cf. (28)) for the semiuniform triangulation of Section 3. Thus, all the analysis in Section 3, and in particular Theorem 3.1, apply to the modified method.

Acknowledgment. I wish to thank Richard Falk for many beneficial discussions and for the improvements he suggested in this manuscript.

Department of Computer Science

Rutgers University

New Brunswick, New Jersey 08903

1. R. S. FALK \& G. R. RICHTER, "Analysis of a continuous finite element method for hyperbolic equations," SIAM J. Numer. Anal., v. 24, 1987, pp. 257-278.

2. C. JOHNSON \& J. PITKÄRANTA, "An analysis of the discontinuous Galerkin method for a scalar hyperbolic equation," Math. Comp., v. 46, 1986, pp. 1-26.

3. C. JohnSON \& J. PITKÄRANTA, "Convergence of a fully discrete scheme for two-dimensional neutron transport," SIAM J. Numer. Anal., v. 20, 1983, pp. 951-966.

4. P. LESAINT \& P. A. RAVIART, "On a finite element method for solving the neutron transport equation," in Mathematical Aspects of Finite Elements in Partial Differential Equations (C. deBoor, ed.), Academic Press, New York, 1974, pp. 89-123.

5. W. H. REed \& T. R. HILL, Triangular Mesh Methods for the Neutron Transport Equation, Los Alamos Scientific Laboratory Report LA-UR-73-479. 